\title{
EchoGéo
}

$19 \mid 2012$

Du littoral à la haute mer : quelles recherches récentes en géographie?

\section{Entretien avec Christian Huetz de Lemps autour de l'ouvrage Comme un parfum d'îles. Florilège offert à Christian Huetz de Lemps}

Marie Redon

\section{OpenEdition}

\section{Journals}

Édition électronique

URL : https://journals.openedition.org/echogeo/12820

DOI : $10.4000 /$ echogeo. 12820

ISSN : 1963-1197

Éditeur

Pôle de recherche pour l'organisation et la diffusion de l'information géographique (CNRS UMR 8586)

Référence électronique

Marie Redon, «Entretien avec Christian Huetz de Lemps autour de l'ouvrage Comme un parfum d'îles. Florilège offert à Christian Huetz de Lemps », EchoGéo [En ligne], 19 | 2012, mis en ligne le 10 février 2012, consulté le 11 août 2021. URL : http://journals.openedition.org/echogeo/12820 ; DOI : https:// doi.org/10.4000/echogeo.12820

Ce document a été généré automatiquement le 11 août 2021.

EchoGéo est mis à disposition selon les termes de la licence Creative Commons Attribution - Pas d'Utilisation Commerciale - Pas de Modification 4.0 International (CC BY-NC-ND) 


\title{
Entretien avec Christian Huetz de Lemps autour de l'ouvrage Comme un parfum d'îles. Florilège offert à Christian Huetz de Lemps
}

\author{
Marie Redon
}

1 L'entretien a été réalisé le vendredi 4 novembre 2011 à l'Institut de Géographie, Paris. Le fil de l'entretien suit celui de l'ouvrage ${ }^{1}$, découpé en quatre grandes parties, comprenant pour certaines des sous-thèmes. Chacun des grands thèmes évoqués par les contributeurs de l'ouvrage donne ici lieu à une question adressée à Christian Huetz de Lemps.

2 - Marie Redon (MR). La première partie, «des découvertes aux utopies, les expérimentations insulaires », s'ouvre sur la question des «îles de l'imaginaire » ( $p$ 33-65). A l'heure où se multiplient les atlas, jusqu'à un Atlas des îles abandonnées, où la cartographie est devenue omniprésente, quels rapports établissez-vous entre imaginaire des îles et cartes ? Peut-on développer un imaginaire insulaire sans atlas et sans cartes marines?

3 - Christian Huetz de Lemps (CHL). Les îles utopiques sont en soi un imaginaire, c'est le seul cas où l'on peut se passer de la carte. Il existe beaucoup d'utopies situées dans les îles mais qui ne s'accompagnent pas forcément de cartes. Certains ouvrages cités dans l'introduction de Comme un parfum d'îles, ou bien d'autres ouvrages sur lesquels j'ai travaillé au sujet des terres australes au XVII ${ }^{e}$ siècle, en large partie insulaires, n'ont pas d'appareil cartographique. Parfois, les auteurs ont ajouté des cartes pour rendre leurs écrits plus crédibles, mais dans ces utopies, ce n'est pas la géographie qui est en cause, ce sont plutôt des utopies sociales que géographiques.

Il existe également tout un imaginaire d'îles bel et bien cartographiées qui parsèment les océans depuis le Moyen-âge. Les grands découvreurs sont partis vers ces îles hypothétiques, comme Christophe Colomb qui comptait s'en servir de relais vers la 
Chine puisqu'il s'imaginait aller vers l'île de Cipango, l'île des Sept Evêques, ou encore Antillas dont le nom existe avant même la découverte des Antilles.

5 De nombreuses îles se sont surajoutées à la réalité au fil du temps, des îles aperçues, entrevues, mais que l'on ne parvenait pas à localiser puisque l'on ne maitrise le calcul de la longitude que depuis les années 1760 ; on cartographiait donc parfois la même île plusieurs fois sur la même carte. Mais ce sont alors de fausses îles plutôt que des îles imaginaires.

6 - MR. Il est ensuite question, via des contributions concernant l'espace asiatique, de «creusets insulaires» (p.69-139) mais qu'en est-il de l'effet de serre sur les îles, paradigme à la fois de l'isolement et de la promiscuité ?

7 - CHL. C'est un élément fondamental de l'insularité que ce phénomène de resserrement. Il y a tout un débat sur les spécificités insulaires, on se demande même si les îles existent... J'ai toujours pensé qu'elles existaient et qu'il y avait une spécificité dans leur perception par les insulaires eux-mêmes, à côté de celle des continentaux qui ont leur propre vision des îles. Les insulaires ont une perception différente de l'espace et du temps: sans faire de déterminisme élémentaire, le milieu influe sur l'homme et l'isolement, l'enfermement insulaire, est un élément qui peu modifier l'approche de l'espace et du temps.

8 Ma première perception de l'originalité des mondes insulaires ne vient pas d'îles tropicales ; c'est en faisant ma maîtrise sur l'île d'Yeu que j'ai commencé à travailler sur les îles. Cette île a toutes les spécificités de l'insularité avec l'ambigüité de l'ouverture et de la fermeture qui est caractéristique des îles. Je me suis beaucoup intéressé à l'histoire de cette île qui a été le plus grand port de cabotage de tout le littoral atlantique français pendant plus de deux siècles. Des centaines de caboteurs, au minimum 250 caboteurs, faisaient la navette entre Bordeaux, la Bretagne, l'Irlande, les pays du Nord pour transporter du vin de Bordeaux. Il s'agit donc d'un remarquable cas d'ouverture. Mais, en même temps, quand on étudie la démographie de l'île d'Yeu, c'est un monde parfaitement endogame, fermé, qui se dessine, avec dix patronymes qui représentaient plus de $80 \%$ de la population. Toujours cette dialectique de l'ouverture et de la fermeture...

9 Du point du vue du rapport à l'espace, alors que l'île avait une petite production de blé, on y trouvait un extrême morcellement du parcellaire qui montrait un extraordinaire attachement à la terre, attachement qui est, à mon avis, caractéristique des petits mondes insulaires avec ce besoin de se raccorder à quelque chose de stable. Peut-être que je caricature, mais il est tout à fait remarquable de voir ce parcellaire, avec des milliers de parcelles qui font parfois 3 mètres de large et $50 \mathrm{~cm}$ de long, et qui témoignent donc d'un émiettement qui contraste totalement avec le parcellaire continental des régions vendéennes à quelques kilomètres de là.

10 Là, dans le cas de l'île d'Yeu, il n'y a pas eu d'immigration mais en revanche un phénomène d'émigration. Les migrations jouent un rôle très important dans la vie des îles. Les diasporas insulaires sont des éléments extrêmement importants. J'ai notamment étudié le cas des Samoans aux États-Unis, en Nouvelles Zélande, etc. avec toujours cette ambigüité de la volonté de partir et du désir de revenir et de garder des liens. Ce n'est peut-être pas spécifique aux insulaires mais c'est particulièrement net chez les insulaires, avec cette volonté de garder ce qui, pour eux, fait l'identité de l'île, dans le domaine culturel, culinaire par exemple. Je me souviens de ces avions qui partaient de Pago Pago, capitale des Samoa américaines, et qui allaient vers les 
communautés samoanes installées en Californie et aux Hawaï. On chargeait les carlingues de ces avions de petits tonnelets de corned-beef néo-zélandais parce que c'était un élément devenu indispensable à la cuisine samoane alors que, bien sûr, on trouve du corned-beef très facilement sur place !

11 Il y a donc, par ces phénomènes migratoires, des superpositions de populations avec un problème de mélanges, de contacts, à l'intérieur d'un espace resserré. Il n'y a pas que dans les îles que cela peut poser problème mais disons que dans un milieu confiné, les fermentations peuvent se produire plus vite. J'ai beaucoup étudié le cas des Hawaï qui est un cas de construction d'une société pluriethnique que je qualifierais d'harmonieuse. Certes, il y a des petites tensions mais c'est globalement harmonieux contrairement aux Fidji où il y a des affrontements entre ceux qu'on appelle "Fidjiens", les Mélanésiens, et les descendants d'Indiens venus travailler sur les plantations de cannes à sucre. On pourrait dire que l'on retrouve ces phénomènes de tensions dans les milieux urbains.

12 - MR. La deuxième partie de l'ouvrage est consacrée aux « îles dans la mondialisation », avec des contributions allant de l'étude des comptoirs à celle des resorts. On assiste actuellement à une course aux espaces maritimes, à l'appropriation de Zones économiques exclusives et des ressources qu'elles contiennent. Dans le prolongement des étapes de l'ouverture des îles au monde (p. 137-210), comment voyez-vous le rôle des îles dans cette nouvelle donne géopolitique?

13 - CHL. Il y a plusieurs échelles à considérer. Il y a, d'un côté, les grands archipels, que l'on peut traiter au même niveau que des puissances continentales, et, de l'autre, les petits États insulaires indépendants. Dans ce cas, tout dépend de leur situation par rapport aux grand flux d'hommes, de marchandises, etc. Il y a des îles qui sont susceptibles d'acquérir une importance disproportionnée par rapport à leur taille, leur population, en raison de leur situation.

14 En revanche, je crois que si l'on exclut les petites îles qui deviennent des destinations touristiques, certaines peuvent être mises à l'écart de la mondialisation parce qu'elles ont perdu l'utilité d'escale qu'elles ont eues. Certaines petites îles ont ainsi joué un très grand rôle puis sont retombées dans l'anonymat lorsque les avions ont été capables de traverser le Pacifique. J'ai par exemple atterri en 1964 sur l'îlot de Wake qui a joué un grand rôle dans le Pacifique Nord parce que, situé à peu près à mi-distance entre Hawaï et le Japon, il a servi d'escale aérienne puis est retombé dans un total anonymat avec l'avènement des «jets » à long rayon d'action Il y a tout un ensemble d'îles et d'archipels qui ont ainsi perdu une partie de leur importance.

Les Samoa américaines ont été ainsi été acquises par les Américains parce qu'elles avaient une position stratégique qui leur semblait essentielle dans le Pacifique, avec la belle rade de Pago Pago. Cette partie orientale des Samoa a donc été gérée par l'US Navy, puis on s'est rendu compte que ça n'avait plus d'intérêt, après la Seconde Guerre mondiale, et on a substitué aux bases des conserveries de thon. L'US Navy s'est alors retirée et a emmené avec elle plusieurs milliers de Samoans qui travaillaient sur la base de Pago Pago et sont allés s'installer aux Hawaï.

Donc là encore, c'est du cas par cas, mais c'est toujours du cas par cas avec les îles. C'est un sujet de discussion avec les amis et collègues que cette singularité, cette diversité des îles, qui fait que je trouve très intéressant, très utile, de comparer les îles, de faire 
des typologies des îles mais définir un modèle insulaire, c'est pour moi une schématisation qui n'apporte pas grand-chose et conduit même à des contresens.

Je commençais toujours mon cours à partir de ce modèle récurrent de l'île tropicale, reproduit un peu partout et qui est une hérésie totale dans le Pacifique par exemple. D'abord, parce que la vraie île tropicale, celle qui n'existe que sous les tropiques, c'est l'atoll. Il faudrait donc préciser que c'est un modèle d'île haute. D'autre part, situer la petite agriculture indigène sous le vent, et l'occupation européenne au vent est contraire à la réalité dans tout le Pacifique parce que au vent, c'est le versant où les précipitations sont suffisantes et donc tous les systèmes traditionnels, fondés sur les racines, les tubercules, les cocoteraies, etc. prospèrent au vent alors que c'est trop sec sous le vent. A l'inverse, les Européens ont développé leurs plantations sous le vent parce qu'il n'y avait pas d'indigènes et parce qu'ils avaient les moyens techniques de développer l'irrigation. Et puis il y a un fort ensoleillement. La règle dans le Pacifique est donc l'inverse. C'est aussi vrai pour la Nouvelle-Calédonie où les concentrations de Kanak sont au vent alors que les Broussards, enfin ce qu'il en reste, sont sous le vent.

18 - MR. Au sujet de l'aménagement des îles (p. 210-247), il est question des îles artificielles des Emirats Arabes Unis : sont-elles des îles à vos yeux ? Est-ce la mer ou l'homme qui fait l'île?

19 - CHL. Ce sont des îles-plaisanteries... ça n'a pas valeur d'île. De toute façon, il y a toujours ce pédoncule qui les rattache à la terre, on pourrait parler de presqu'îles à la limite. Il y a des îles artificielles auxquelles on n'accède que par bateau, comme dans la barrière corallienne des Salomon où de petites îles ont été construites par l'homme et sont habitées par des populations qui avaient peur des razzias des bushmen venant de l'intérieur. Mais ce sont des îlets.

20 L'insularité est quelque chose qui se construit, qui s'imprime dans la mentalité. Les îlespalmiers de Dubaï, je refuse de les classer dans les îles!

21 - MR. La « voie touristique » fait partie des moyens par lesquels les îles sont arrimées à la mondialisation (p. 251-334); vous considérez-vous aussi comme un touriste, comme un consommateur d'îles? Quelles sont les îles où vous rêvez d'aller?

22 - CHL. Oui, je suis touriste, bien sûr, en ce sens que je m'émerveille facilement de la beauté des îles, mais j'en profite pour travailler aussi... Nous sommes des consommateurs d'îles, mais on peut être consommateur sans être destructeur.

23 J'aimerais me rendre sur toutes les îles que je ne connais pas! C'est François Doumenge qui disait que l'on ne peut parler des îles que si on les connait toutes, précisément en raison de cette diversité intrinsèques aux îles. Et il est celui que je connais qui a parcouru le plus d'îles. Toutes les îles m'intéressent, mais il y des îles plus faciles, plus agréables à "consommer » que d'autres. Par exemple, la Nouvelle-Guinée, j'en rêve mais elle n'est pas facile à "consommer». C'est un ensemble insulaire avec des chapelets d'îles prodigieux. Je m'étais régalé aux Salomon, c'est extraordinaire, on a l'impression d'y retrouver le Pacifique du XIX ${ }^{\circ}$ siècle, c'est-à-dire de retrouver le Pacifique d'avant les Européens, pratiquement.

24 Il y a de grosses îles que je ne connais pas et que j'aimerais connaitre, comme le Sri Lanka, Haïti et la République dominicaine....

25 - MR. Le tourisme est parfois accusé d'accroitre la vulnérabilité des îles. Le rapport « îles et vulnérabilité » (p. 337-395) est mis en évidence par toute une littérature 
catastrophiste dont les petits espaces insulaires semblent être la cible privilégiée. Doiton être inquiet pour les îles du Pacifique?

- CHL. Nous n'avons pas beaucoup de recul, les catastrophes sont pour l'instant plus liées à des phénomènes ponctuels qu'à une véritable évolution sur le long terme. Il y a des îles qui sont particulièrement menacées, ce sont les îles basses, les atolls, comme les Tuvalu ou les Maldives si le niveau de la mer continue à monter. Il est indéniable que le niveau a monté mais jusqu'où et quelles en sont les véritables causes? Tout cela est encore un peu flou.

27 Mais, pour les îles, la menace ne porte que sur des chiffres de population très faibles. La véritable menace c'est le Bangladesh, c'est la Thaïlande, toutes ces terres basses des littoraux, ces deltas où les conséquences peuvent être considérables sur le plan humain. Tuvalu ne représente que 12000 habitants. Certes, il y a un risque mais ce n'est pas un risque nouveau. Les Tuamotu, en 1903, ont par exemple été balayées par une série de cyclones qui ont fait pratiquement disparaitre certaines îles, qui les ont raclées jusqu'au platier corallien; c'est inhérent aux îles basses.

28 Si l'on doit faire du catastrophisme, ce ne sont pas les réfugiés de Tuvalu qui vont modifier l'équilibre démographique mondial. Je trouve que c'est une forme d'instrumentalisation, on n'a jamais tant parlé du Tuvalu que ces derniers temps! Mais les vrais problèmes sont davantage à la Nouvelle Orléans ou au Bangladesh que dans les espaces insulaires. A l'échelle mondiale, nous sommes désormais 7 milliards. Mais, là aussi, il faut être prudent sur les prévisions démographiques, on annonçait 20 milliards d'êtres humains pour 2050 dans les années $1960 . . .0$ ne dépassera pas 10 selon toutes probabilités Ce catastrophisme insulaire est surtout révélateur d'une crainte générale.

29 - MR. L'ouvrage se clôt sur les notions d'insularisme et d'insularité (p. 399-485). Peut-on échapper aux îles, en tant que géographe, quand on a commencé à travailler sur ce thème? Ne porte-t-on pas ensuite un regard de chercheur « insularisé » sur le monde, les îles devenant une sorte de prisme de lecture?

30 - CHL. Non, je ne crois pas, j'ai fait beaucoup de coopération avec des universités africaines en partenariat avec Bordeaux III, notamment avec sept universités africaines situées dans des pays enclavés, alors oui, j'ai parfois vu des analogies entre enclavement et insularité... Mais en dehors de cela, on peut avoir un œil neuf, non pollué par l'insularité.

31 On n'est pas prisonnier en tant que chercheur mais dans une île, oui, on peut être prisonnier. L'insularisme, terme qui n'est pas français d'ailleurs, est un problème qui fait partie des conséquences humaines de l'insularité, c'est cette tendance à tout juger à l'aune de l'île mais il faut être insulaire pour cela. Mes centres d'intérêt sont variés, même si j'ai consacré beaucoup de temps aux îles. J'ai consacré au moins autant de temps à l'histoire qu'à la géographie, j'ai beaucoup travaillé sur des problèmes commerciaux. J'ai par exemple rédigé un livre sur le commerce à Bordeaux à la fin du règne de Louis XIV, beaucoup dépouillé d'archives, alors non, je ne me sens pas prisonnier des îles. Il y a beaucoup de choses que j'ai envie de faire qui sont en rapport avec les îles, comme approfondir mes études sur les Engagés, ces pauvres Blancs recrutés par contrats notariés de 36 mois, en complétant les dépouillements effectués dans les archives de Bordeaux.

32 Nous sommes marqués par nos terrains principaux de recherche. Je crois que le géographe des îles est quasiment obligatoirement un géographe de terrain, et il est 
presque obligatoirement aussi un géographe de synthèse parce qu'il y a vraiment des systèmes insulaires à saisir.

On continue à garder des liens avec ses terrains; Guy Lasserre était l'homme de la Guadeloupe, Jean-Claude Giacottino était l'homme de Trinidad et Tobago, même s'ils ont fait bien autre chose. Cela me parait normal, sans être signe d'un enfermement. En général, après la thèse d'Etat, ces chercheurs élargissaient leurs horizons.

- MR. On voit bien l'ampleur et la densité de ce que les îles vous ont apporté et vous apportent, mais que pensez-vous apporter aux îles, vous?

- CHL; La question est difficile... Je n'ai pas fait d'humanitaire dans les îles... Mais peutêtre qu'on contribue à les protéger un petit peu de l'insularisme, oui, c'est en cela qu'on peut être utile. Je viens de faire soutenir une série de thèses sur la Nouvelle-Calédonie et et j'y ai décelé ici et là certains éléments de repli sur soi qui sont typiquement insularistes.

On peut aussi être utile en tant que truchement entre insulaires et continentaux parce que les visions sont souvent simplistes des îles. Les mondes insulaires sont très mal connus, notamment le Pacifique. Les gens confondent Hawaï et Haïti, Haïti et Tahiti... Nous avons un rôle de passeurs.

\section{NOTES}

1. Sevin O., Chaléard J.-L, Guillaud D. (dir), 2010. Comme un parfum d'îles. Florilège offert à Christian Huetz de Lemps. PUPS, $522 \mathrm{p}$.

\section{AUTEUR}

\section{MARIE REDON}

Marie Redon, mredon@yahoo.com, est Maître de conférences à l'Université Paris 13-Nord. Elle a publié récemment : Redon M., 2010. Des îles en partage. $326 \mathrm{p}$ 